\title{
Differences in Elastin and Elastolytic Enzymes between Men and Women with Abdominal Aortic Aneurysm
}

\author{
Christina Villard, $\mathrm{MD}^{1,2 *}$, Per Eriksson, $\mathrm{PhD}^{3}$, Jesper Swedenborg, $\mathrm{PhD}^{1}$, \\ Rebecka Hultgren, $\mathrm{MD}, \mathrm{PhD}^{1,2}$ \\ ${ }^{1}$ Department of Molecular Medicine and Surgery, Karolinska Institutet, Stockholm, Sweden; ${ }^{2}$ Department of Vascular Surgery, \\ Karolinska University Hospital, Stockholm, Sweden; and ${ }^{3}$ Atherosclerosis Research Unit, Center for Molecular Medicine, Department \\ of Medicine, Karolinska Institutet, Stockholm, Sweden
}

\begin{abstract}
Background: Abdominal aortic aneurysms (AAAs) in women differ in some important aspects from those in men. The lower prevalence rate, higher rupture rate and potentially increased growth rate in women with AAA suggest gender to be of importance for aneurysm development and progression. The aim of the study was to analyze wall properties with respect to synthesis and destruction of elastin in men and women with AAA, with and without an intraluminal thrombus. Methods: Patient characteristics and aneurysm wall biopsies were collected from all women $(n=14)$ treated with open repair for AAA, 2008-2012, and men with similar aneurysm diameter and similar age $(n=23)$ treated during the same time period. The expressions of elastin, matrix metalloproteinase (MMP)-2 and -9, and cathepsin K were quantified by immunohistochemistry, Western blot, and gene expression analysis on the aneurysm wall. Results: The protein expression of elastin was less in women than in men in the non-thrombus-covered aneurysm wall. In addition, the protein and mRNA expressions of MMP-9 were higher in women $(-0.83$ versus 0.09 , $P=0.041)$. There was no difference in elastin and elastolytic enzymes between men and women in the thrombus-covered aneurysm wall. Conclusion: Less elastin in the non-thrombus-covered aneurysm wall in women than that in men, and the simultane-
\end{abstract}

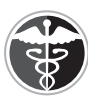

Fax +1 2037853346

E-Mail: aorta@scienceinternational.org

http://aorta.scienceinternational.org (c) 2014 Aorta

Published by Science International Corp. ISSN 2325-4637

Accessible online at:

http://aorta.scienceinternational.org ous higher level of MMP-9, suggest differences in the elastolytic process in AAA between the sexes.

Copyright $\odot 2014$ Science International Corp.

\section{Key Words}

Abdominal aortic aneurysm - Gender - Women - Elastin - MMP-9 • Progression - Rupture

\section{Introduction}

Abdominal aortic aneurysms (AAAs) in women differ in some important aspects from those in men. The lower prevalence rate, higher rupture rate and potentially increased growth rate in women with AAA suggest gender to be of importance for aneurysm development and progression. [1-3].

The development of AAA is characterized by the loss of elastin in the medial layer of the aortic wall together with an increased collagen turnover $[4,5]$. With the depletion of elastin and collagen the aorta loses its elasticity and tensile strength [6]. Elastin, which is synthesized by vascular smooth muscle cells (VSMCs) in the medial layer of the aortic wall, is composed to impart resilience for a lifetime $[7,8]$.

The breakdown of elastin in AAA development is induced by proteolytic enzymes, produced by infil-

*Corresponding author:

Christina Villard, MD

Department of Vascular Surgery

Karolinska University Hospital

17176 Stockholm, Sweden

Tel: +001 4685177 2288, Fax: +0046871776642, E-Mail: christina.villard@ki.se 
trating inflammatory cells and modified VSMCs [9]. The family of matrix metalloproteinases (MMPs) are the proteolytic enzymes most associated with AAA development [10]. Especially MMP-2 and -9, with substantial elastolytic capacity, contribute to both AAA development and growth $[11,12]$. The family of cathepsins is another group of matrix-degenerating enzymes inducing elastolysis that has been implicated in the formation and growth of AAA [13].

The intraluminal thrombus (ILT), often prevalent in AAA, has been show to influence the deterioration of the underlying aneurysm wall [14]. Beneath the ILT the fragmentation of elastic fibers is more pronounced, the vessel wall is thinner, and there are more inflammatory cells and fewer VSMCs than in the nonthrombus-covered wall $[14,15]$. The effect mediated by the ILT has been related to its thickness and size; a thin ILT has been shown to be more biomechanically active and to more effectively promote proteolysis in the underlying aneurysm wall, whereas a thicker ILT contributes to the deterioration of the underlying aneurysm wall by inducing hypoxia $[16,17]$.

Women have smaller aortas than men, rendering the relative enlargement in women with AAA greater compared to that of men at any given diameter, but little is known of how it relates to the deterioration of the aneurysm wall $[18,19]$. Aortic size index (ASI) is a measurement taking the relative dilatation into consideration [20]. Gender differences in vascular aging have been ascribed to the effect of sex hormones [21]. Differences between the sexes in the degradation of the arterial wall, induced by senescence, have been illustrated by the maintenance of elasticity in the aorta of women compared to that of men [22]. Thereto, estrogen has been shown to increase the deposition of elastin by VSMCs [23].

A difference in elastin and elastolytic proteins between men and women with AAA could help explain the gender disparities in AAA progression. The aim of the study was to compare elastin content and some of the elastolytic enzymes mostly associated with AAA development, between men and women with equally large AAA.

\section{Materials and Methods}

All women treated electively for AAA with open aneurysm repair at Karolinska University Hospital, November 2008 to
December 2012, were included $(n=14)$. Male AAA patients within similar age and aneurysm diameter, treated during the same time period, were also included $(n=23)$. The patients were treated with open repair (OR) either because they were deemed unfit for endovascular aneurysm repair (EVAR) or because of their comparatively young age. A biopsy of the ventral infrarenal aneurysm wall, preferably in midline, often at the maximum diameter, was obtained during operation. Two biopsies were taken from each patient, if possible: thrombuscovered and non-thrombus-covered aneurysm wall. The thrombus was removed and stored separately.

Patient characteristics were obtained from hospital charts. Body surface area (BSA) was calculated according to DuBois: body surface area $\left(\left(\right.\right.$ weight $^{0.425} \times$ height $\left.\left.\left.^{0.725}\right) \times 0.007184\right)\right)$ [24]. ASI was calculated: aneurysm diameter $(\mathrm{cm}) / \mathrm{BSA}\left(\mathrm{m}^{2}\right)[20]$.

All patients had signed an informed consent prior to the surgical procedure. The study was approved by the local Ethics Committee.

\section{Immunohistochemistry}

Sections $(5 \mu \mathrm{m})$ of thrombus-covered and non-thrombuscovered aneurysm walls were deparaffinized in Tissue-Clear ${ }^{\mathrm{TM}}$ (Sakura, Leiden, The Netherlands) and rehydrated in ethanol. Weigert-van Gieson staining was then performed by immersing the sections in the various solutions: Weigert's hematoxylin, Weigert's elastin, and Van Gieson solutions, all from SigmaAldrich (St. Louis, Missouri, USA), according to a standardized protocol. Elastic fibers turned dark purple, muscle turned yellow, nuclei turned brown, and connective tissue turned red. The investigator was blinded for sex and AAA diameter when valuing the elastin staining.

\section{Gene Expression Analysis}

Snap-frozen thrombus- and non-thrombus-covered media were homogenized in Lysing Matrix D tubes with Fastprep ${ }^{\circledR}$ (MP Biomedicals, Solon, Ohio, USA). RNA was isolated with TRIzol (Life Technologies, Grand Island, New York, USA), RLT buffer (from RNeasy Mini kit, Qiagen, Hilden, Germany) and DNase1 (RNase free DNase Set, Qiagen) according to the manufacturer's protocol. RNA was quantified by a NanoDrop (NanoDrop Products, Wilmington, Delaware, USA), and RNA quality and integrity were verified using the Agilent 2100 Bioanalyzer System (Agilent Technologies, Santa Clara, California, USA).

For quantification of gene expression, total RNA, was reverse-transcribed to cDNA using Superscript II according to the manufacturer's protocol (Life Technologies). Real-time polymerase chain reaction (PCR) was performed on the Applied Biosystems (Foster City, California, USA) 7000 Real-Time PCR System with TaqMan Assays-on-Demand Gene Expression Probes for elastin, MMP-9, MMP-2, and cathepsin K. Robust multiarray average normalization was performed and gene expression data were log2-transformed. The housekeeping gene Ribosomal Protein Large PO (RPLPO) was used for normalization.

\section{Western Blot}

Thrombus- and non-thrombus-covered medial layers were shred and put in tubes with a lysis buffer containing 50 pro- 
tease inhibitor and $301 \mathrm{~mol} / \mathrm{L}$ Tris- $\mathrm{HCl}, \mathrm{pH}$ 8.0. The samples were then granulated with a Qiagen Tissuelyser according to the manufacturer's protocol, followed by centrifugation for 5 minutes at $220 \mathrm{rpm}$. The supernatants were sonicated for 5 minutes at high level followed by centrifugation for 10 minutes at $12,000 \mathrm{rpm}$. The protein content in the supernatants was measured using a Bradford protein assay.

The protein was diluted with lysis buffer and then loaded on a $4-12 \%$ sodium dodecyl sulfate (SDS) gel [Novex NuPAGE ${ }^{\oplus}$ 4-12\% Bis-Tris gel, 15 well (Life Technologies)] in MOPS-SDS running buffer. The electrophoresis was performed in a cold room for 90 minutes at $100 \mathrm{~V}$. Before transfer by electroblotting in a cold room for 90 minutes at $400 \mathrm{~mA}$, the gel and membrane (Hybond Polyvinylidene Flouride transfer membrane, GE Healthcare, Little Chalfont, Buckinghamshire, United Kingdom) were equilibrated in transfer buffer. For blocking, the membrane was suspended in blocking buffer (3\% bovine serum albumin/Tween Tris-buffered saline) for 60 minutes. The membrane was incubated overnight with elastin (Abcam, Cambridge, United Kingdom), MMP-9 (Cell Signaling Technology, Danvers, Massachusetts, USA), $\beta$-tubulin (Santa Cruz Biotechnology, Santa Cruz, California, USA), and Glyceraldehyde 3-phosphate dehydrogenase (Abcam) followed by the second antibody (anti-mouse and anti-rabbit HRP, Bio-Rad Laboratories, Hercules, California, USA) for 45 minutes. For the final step of chemiluminescent detection, the developing solution from ECL Prime Western Blotting Detection Reagent kit (GE Healthcare) and film (Amersham Hyperfilm ECL, GE Healthcare) were used. Densitometry was performed using ImageJ analysis software.

\section{Statistics}

The statistical analysis was performed with SPSS 21.0. The independent $t$ test was used for gender comparisons of normally distributed data and the Mann-Whitney $U$ test for continuous, not normally distributed, data. Pearson's $\chi^{2}$ test and Fisher's exact test were used for parametric and nonparametric categorical variables, respectively. Statistical significance was defined as $P<0.05$.

\section{Results}

\section{Body Surface Area and Aortic Size Index in Men and Women with $A A A$}

Men and women with AAA were similar in age and had similar aneurysm diameter and body mass index (BMI). Men with AAA had higher BSA than women with $A A A, 1.9$ versus $1.7, P<0.001$. There was no difference in the absolute diameter relative to $B S A$ $(A S I)$, i.e., AAA diameter $(\mathrm{cm}) / B S A\left(\mathrm{~m}^{2}\right)$, between men and women (Table 1). Men and women with AAA were similar regarding smoking habits, occurrence of comorbid conditions, relevant laboratory parameters, and medical treatments, with the exception of beta blockers, which was more frequently prescribed to women (Table 1).

\section{The Non-Thrombus-Covered Aneurysm Wall in Men and Women}

Western blot analysis and immunohistochemistry showed that protein expression of elastin in the nonthrombus-covered aneurysm wall was lower in women than in men (Fig. 1) There was no difference in mRNA expression of elastin between men and women (Table 2). Women had a higher mRNA expression of MMP-9 than men (Table 2). Similar results were observed in Western blot analysis (Fig. 1). There were no differences in MMP-2 and cathepsin $\mathrm{K}$ between men and women in mRNA expression analysis (Table 2). Prior and current smokers as well as smoking and non-smoking men and women were similar in the mRNA expressions of elastin, MMP-9 and cathepsin K.

\section{The Thrombus-Covered Aneurysm Wall in Men and Women}

There was no difference in mRNA expression or protein expression of elastin, MMP-2, MMP-9, and cathepsin $\mathrm{K}$ in the thrombus covered aneurysm wall between men and women (Table 3). There were no differences in mRNA expressions of elastin, MMP-2 and -9 , and cathepsin $\mathrm{K}$ between prior and current smokers or between smoking and nonsmoking men and women.

\section{Discussion}

The key findings of this study, less elastin in the non-thrombus-covered aneurysm wall of women compared to that of men with a simultaneous greater expression of MMP-9, imply that the elastolytic process in the aneurysm wall differs between the sexes.

Elastin breakdown has been shown to be of importance especially in the initial growth of an $A A A$, whereas collagen degradation has been associated with the later stages of aneurysm enlargement and rupture [6]. Less protein expression of elastin in the non-thrombus-covered aneurysm wall of women than in men, together with the opposite relation in protein and mRNA expression of MMP-9, suggests a more elastolytic process in that part of the aneurysm wall of women. The lack of difference in elastin content and proteolytic enzymes in the thrombus-covered aneurysm wall between the sexes, found in this study, 
Table 1. Patients' Characteristics

\begin{tabular}{|c|c|c|c|}
\hline & Men with $\mathrm{AAA}(N=23)$ & Women with AAA $(N=14)$ & $P$-value \\
\hline Age & $71 \pm 5$ & $72 \pm 7$ & 0.595 \\
\hline AAA diameter & $6.3 \pm 0.9$ & $6.0 \pm 0.6$ & 0.263 \\
\hline BMI & $26.0(5.2)$ & $25.0(3.0)$ & 0.752 \\
\hline BSA & $1.9(0.2)$ & $1.7(0.2)$ & $<0.001$ \\
\hline ASI & $3.2 \pm 0.5$ & $3.5 \pm 0.3$ & 0.107 \\
\hline \multicolumn{4}{|c|}{ Risk factors and comorbid conditions } \\
\hline Smoking & & & 0.172 \\
\hline Current & $12(52)$ & $4(29)$ & \\
\hline Prior & $10(44)$ & $9(64)$ & \\
\hline Hypertension & $11(55)$ & $8(57)$ & 0.901 \\
\hline Diabetes mellitus & $3(15)$ & $0(0)$ & 0.261 \\
\hline Renal insufficiency & $1(5)$ & $0(0)$ & 1.000 \\
\hline Cardiac disease $\mathrm{a}^{\mathrm{a}}$ & $3(15)$ & $4(29)$ & 0.410 \\
\hline Connective tissue disorder & $1(5)$ & $1(8)$ & 1.000 \\
\hline \multicolumn{4}{|l|}{ Medical treatment } \\
\hline Beta blockers & $4(21)$ & $10(72)$ & 0.004 \\
\hline Antiplatelet & $15(75)$ & $7(50)$ & 0.163 \\
\hline Statins & $12(60)$ & $8(57)$ & 0.868 \\
\hline \multicolumn{4}{|l|}{ Laboratory values } \\
\hline Albumin ${ }^{\mathrm{b}}$ & $36 \pm 4$ & $38 \pm 3$ & 0.128 \\
\hline Creatinine ${ }^{b}$ & $86 \pm 19$ & $82 \pm 24$ & 0.614 \\
\hline \multicolumn{4}{|c|}{$\begin{array}{l}\text { Values are presented as mean } \pm \text { standard deviation for normally distributed data, median }(\mathrm{IR}) \text { for not normally distributed data, and frequencies }(\%) \text { for categorical variables. } \\
\text { Significance calculated by independent } t \text { test, Mann Whitney } U \text { test, Pearson's chi-square test, and Fisher's exact test. BMI }=\text { body mass index; BSA }=\text { body surface area } \\
\left.\left(\text { weight }^{0.425} \times \text { height }^{0.725}\right) \times 0.007184\right) \text {; ASI }=\text { aortic size index }\left\{\text { aneurysm diameter }(\mathrm{cm}) / \mathrm{BSA}\left(\mathrm{m}^{2}\right)\right\} \text {. }\end{array}$} \\
\hline \multicolumn{4}{|c|}{${ }^{a}$ Cardiac disease defined as cardiac insufficiency, coronary artery disease, and atrial fibrillation. } \\
\hline bLaboratory values obtained 1 or & e surgery. & & \\
\hline
\end{tabular}

suggest that the elastolysis in that part of the aneurysm wall is similar in men and women. The impact of the limited expression of elastin in the non-thrombuscovered aneurysm wall of women remains to be investigated but could contribute to explaining gender differences in AAA pathogenesis. A weaker part of the aneurysm wall, as a result of greater elastolysis, in women could be of importance for the growth and rupture rate of women's AAAs. The difference in protein expression of elastin between men and women without a simultaneous difference in the mRNA expression could be explained by the limited turnover rate and synthesis of elastin in adults [8]. The similar age and AAA diameter as well as smoking habits of the participating men and women in this study rule them out as possible confounding factors. Tong et al. [19] recently presented results of a higher growth rate in men than in women with AAA and less elastin in men, in the thrombus-covered aneurysm wall. A possible explanation for the contradicting results might be differences in age, AAA diameter, and smoking habits between the participants of the two studies.

MMP-2 has been implicated in the initial AAA development, and higher levels have been observed beneath the ILT [14]. We found no difference in mRNA expression of MMP-2 in men and women either beneath the ILT or in the non-thrombus-covered aneurysm wall. The lack of difference in mRNA expression of MMP-2 and cathepsin K between men and women suggest that the expression of MMP- 2 and cathepsin $\mathrm{K}$ is of less importance for gender differences in AAA progression.

Women have smaller BSA and suprarenal aortic diameter than men, making the relative aneurysm enlargement in women exceed that of men [18]. ASI is a measure-until recently, more commonly used when categorizing thoracic aneurysms - that takes the relative enlargement into account [25]. Lo et al. [20] recently showed that ASI is an important determinant for AAA progression in women. In this 
A)
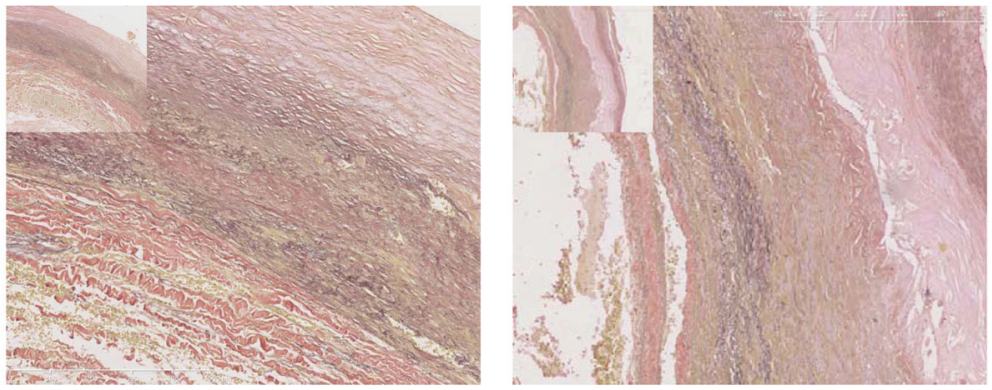

B)
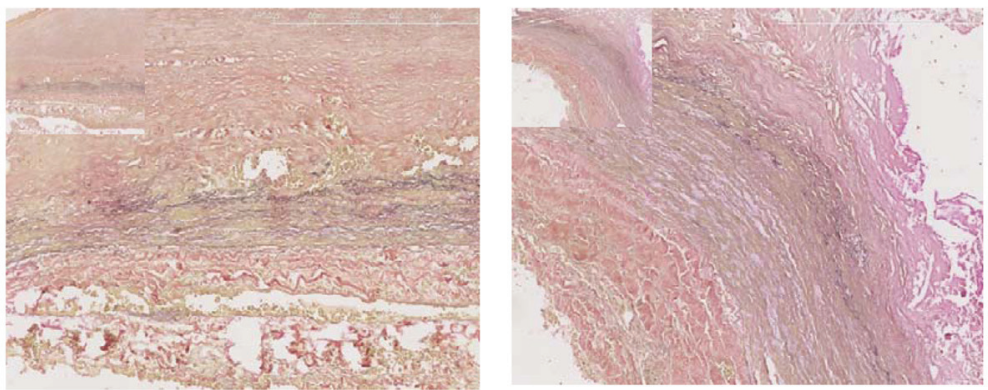

C)
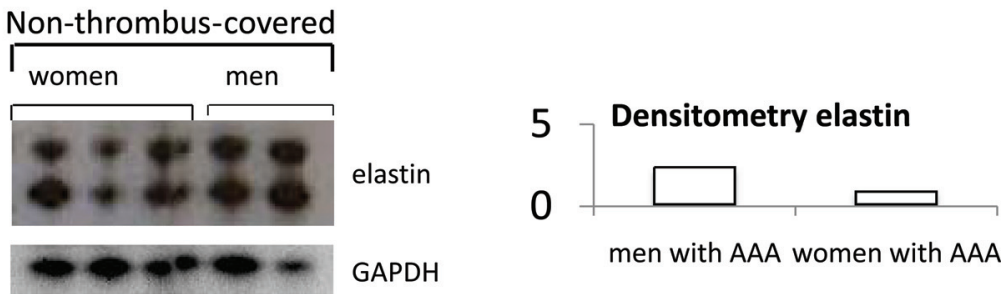

D)
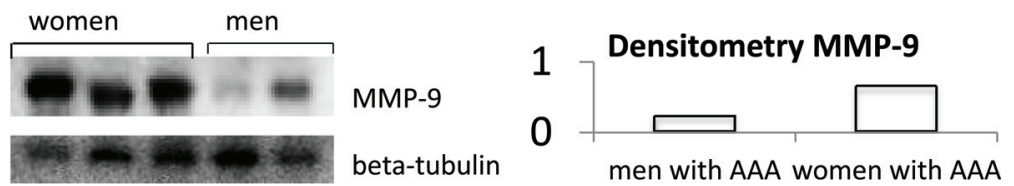

Figure 1. A. Weigert-van Gieson's staining of elastin in the non-thrombus-covered aneurysm wall of two men. B. Weigert-van Gieson's staining of elastin in the non-thrombus-covered aneurysm wall of two women. C. Western blot and densitometry of elastin in the non-thrombus-covered aneurysm wall of three women and two men (0.81 versus $2.30, P=0.200)$. D. Western blot and densitometry of MMP-9 in the non-thrombus-covered aneurysm wall of three women and two men $(0.65$ versus $0.22, P=$ 0.200).

Table 2. Gene Expression Analysis of Elastin, MMP-2, MMP-9, and Cathepsin $\mathrm{K}$ in the Non-Thrombus-Covered Aneurysmal Wall of Men and Women

\begin{tabular}{lrcc}
\hline & \multicolumn{2}{l}{ Non-Thrombus-Covered Wall } & \\
\cline { 2 - 3 } & \multicolumn{1}{c}{$\begin{array}{c}\text { Men } \\
\text { with AAA }\end{array}$} & $\begin{array}{c}\text { Women } \\
\text { with AAA }\end{array}$ & P-value \\
\hline Elastin & $4.55 \pm 3.21$ & $5.01 \pm 3.31$ & 0.722 \\
MMP-2 & $1.52 \pm 1.22$ & $1.74 \pm 1.51$ & 0.744 \\
MMP-9 & $-0.83 \pm 1.08$ & $0.90 \pm 2.59$ & 0.041 \\
Cathepsin K & $1.68 \pm 1.06$ & $2.63 \pm 1.01$ & 0.063 \\
\hline
\end{tabular}

Values are presented as mean \pm standard deviation for normally distributed data. Significance calculated by independent $t$ test. Values are presented log2-transformed and as arbitrary units.
Table 3. Gene Expression Analysis of Elastin, MMP-2, MMP-9, and Cathepsin $\mathrm{K}$ in the Thrombus-Covered Aneurysmal Wall of Men and Women

\begin{tabular}{|c|c|c|c|}
\hline & \multicolumn{2}{|c|}{ Thrombus-Covered Wall } & \multirow[b]{2}{*}{$P$-value } \\
\hline & $\begin{array}{c}\text { Men } \\
\text { with AAA }\end{array}$ & $\begin{array}{c}\text { Women } \\
\text { with AAA }\end{array}$ & \\
\hline Elastin & $-6.03(1.69)$ & $-6.03(1.89)$ & 0.830 \\
\hline MMP-2 & $-2.13 \pm 0.60$ & $-2.41 \pm 0.78$ & 0.282 \\
\hline MMP-9 & $-2.27 \pm 1.08$ & $-2.20 \pm 1.35$ & 0.875 \\
\hline Cathepsin K & $-2.40(1.58)$ & $-2.82(1.89)$ & 0.940 \\
\hline
\end{tabular}

Values are presented as mean \pm standard deviation for normally distributed data and median (interquartile range) for not normally distributed data. Significance calculated by independent $t$ test and Mann Whitney $U$ test. Values are presented log2transformed and as arbitrary units. 
study women were found to have significantly lower BSA than men, but the ASI did not significantly differ between the sexes. We do not rule out that a weaker aneurysm wall, due to a greater enlargement, in women could help explain gender differences in aneurysm development. We cannot, however, conclude that the differences in biomechanical properties found in this study are attributed to such an effect.

There are limitations to this study, of which the small sample size is one. However, the number of patients included in this study is similar to those in other studies within the field of research $[12,15]$. Another limitation to the study is the potential influence of other enzymes involved in the elastolytic process not addressed in this study; for instance, tissue inhibitor of MMP (TIMP). More potentially influential genes could have been found with the use of microarray analysis, for instance. Finally, the biopsies of the aneurysm wall were all obtained from the anterior wall, and consequently the generalizability of the results to the rest of the aneurysm wall cannot be assured.

In conclusion, less elastin in the non-thrombuscovered aortic wall of women with AAA than in men and the simultaneous higher level of MMP-9 suggest differences in the elastolytic process of AAA in men and women.

\section{Conflict of Interest}

The authors have no conflict of interest relevant to this publication.

Comment on this Article or Ask a Question

\section{References}

1. Pleumeekers HJ, Hoes AW, van der Does E, 9. Sakalihasan N, Limet R, Defawe O. Abdomivan Urk $H$, Hofman $A$, de Jong PT, et al. Aneurysms of the abdominal aorta in older adults. The Rotterdam Study. Am J Epidemiol. 1995;142:1291-1299.

2. Mofidi R, Goldie VJ, Kelman J, Dawson ARW, Murie JA, Chalmers RTA. Influence of sex on expansion rate of abdominal aortic aneurysms. Br J Surg. 2007;94:310-314. 10.1002/bjs.5573

3. Sweeting MJ, Thompson SG, Brown LC, Powell JT. Meta-analysis of individual patient data to examine factors affecting growth and rupture of small abdominal aortic aneurysms. Br J Surg. 2012;99:655-665. 10.1002/ bjs.8707

4. Carmo M, Colombo L, Bruno A, Corsi F, Roncoroni L, Cuttin MS, et al. Alteration of elastin, collagen and their cross-links in abdominal aortic aneurysms. Eur J Vasc Endovasc Surg. 2002;23:543-549. 10.1053/ejvs.2002. 1620

5. Dobrin PB, Baker WH, Gley WC. Elastolytic and collagenolytic studies of arteries. Implications for the mechanical properties of aneurysms. Arch Surg. 1984;119:405-409. 10 1001/archsurg.1984.01390160041009

6. Dobrin PB, Mrkvicka R. Failure of elastin or collagen as possible critical connective tissue alterations underlying aneurysmal dilatation. Cardiovasc Surg. 1994;2:484-488.

7. Kielty CM, Sherratt MJ, Shuttleworth CA. Elastic fibres. J Cell Sci. 2002;115:2817-2828.

8. Shapiro SD, Endicott SK, Province MA, Pierce JA, Campbell EJ. Marked longevity of human lung parenchymal elastic fibers deduced from prevalence of D-aspartate and nuclear weapons-related radiocarbon. J Clin Invest. 1991;87:1828-1834. 10.1172/JCI115204 nal aortic aneurysm. Lancet. 2005;365:15771589. 10.1016/S0140-6736(05)66459-8 metalloproteinases in abdominal aortic aneurysms. Ann NY Acad Sci. 1996;800:157174. 10.1111/j.1749-6632.1996.tb33307.x

11. Petersen E, Wågberg F, Angquist KA. Proteolysis of the abdominal aortic aneurysm wall and the association with rupture. Eur J Vasc Endovasc Surg. 2002;23:153-157. 10.1053/ ejvs.2001.1572

12. McMillan WD, Tamarina NA, Cipollone M, Johnson DA, Parker MA, Pearce WH. Size matters: the relationship between MMP-9 expression and aortic diameter. Circulation. 1997;96: 2228-2232. 10.1161/01.CIR.96.7.2228

13. Abdul-Hussien $H$, Soekhoe RG, Weber $E$, von der Thüsen JH, Kleemann R, Mulder A, et al. Collagen degradation in the abdominal aneurysm: a conspiracy of matrix metalloproteinase and cysteine collagenases. Am J Pathol. 2007;170:809-817. 10.2353/ajpath. 2007.060522

14. Koole D, Zandvoort HJ, Schoneveld A, Vink $A$, Vos JA, van den Hoogen $L L$, et al. Intraluminal abdominal aortic aneurysm thrombus is associated with disruption of wall integrity. J Vasc Surg. 2013;57:77-83. 10.1016/j.jvs. 2012.07.003

15. Kazi M, Thyberg J, Religa P, Roy J, Eriksson P, Hedin U, et al. Influence of intraluminal thrombus on structural and cellular composition of abdominal aortic aneurysm wall. J Vasc Surg. 2003;38:1283-1292. 10.1016/S0741-5214(03) 00791-2

16. Wiernicki I, Stachowska E, Safranow K, Cnotliwy M, Rybicka M, Kaczmarczyk M, et al.
10. Thompson RW, Parks WC. Role of matrix
Enhanced matrix-degrading proteolytic activity within the thin thrombus-covered wall of human abdominal aortic aneurysms. Atherosclerosis. 2010;212:161-165. 10.1016/j. atherosclerosis.2010.04.033

17. Vorp DA, Lee PC, Wang DH, Makaroun MS, Nemoto EM, Ogawa $S$, et al. Association of intraluminal thrombus in abdominal aortic aneurysm with local hypoxia and wall weakening. J Vasc Surg. 2001;34:291-299. 10.1067/ mva.2001.114813

18. Forbes TL, Lawlor DK, DeRose G, Harris KA. Gender differences in relative dilatation of abdominal aortic aneurysms. Ann Vasc Surg. 2006;20:564-568. 10.1007/S10016-006-9079-y

19. Tong J, Schriefl AJ, Cohnert T, Holzapfel GA. Gender differences in biomechanical properties, thrombus age, mass fraction and clinical factors of abdominal aortic aneurysms. Eur J Vasc Endovasc Surg. 2013;45:364-372. 10.1016/j.ejvs.2013.01.003

20. Lo RC, Lu B, Fokkema MT, Conrad M, Patel VI, Fillinger $M$, et al. Relative importance of aneurysm diameter and body size for predicting abdominal aortic aneurysm rupture in men and women. J Vasc Surg. 2014;59: 1209-1216. 10.1016/j.jvs.2013.10.104

21. Mendelsohn ME, Karas RH. Molecular and cellular basis of cardiovascular gender differences.Science.2005;308:1583-1587.10.1126/ science. 1112062

22. Astrand H, Stalhand J, Karlsson J, Karlsson M Sonesson B, Länne T. In vivo estimation of the contribution of elastin and collagen to the mechanical properties in the human abdominal aorta: effect of age and sex. J Appl Physiol. 2011;110:176-187. 10.1152/japplphysiol. 00579.2010 
23. Natoli AK, Medley TL, Ahimastos AA, Drew BG, Thearle DJ, Dilley RJ, et al. Sex steroids modulate human aortic smooth muscle cell matrix protein deposition and matrix metalloproteinase expression. Hypertension. 2005;46:11291134. 10.1161/01.HYP.0000187016.06549.96

24. Wang Y, Moss J, Thisted R. Predictors of body surface area. J Clin Anesth. 1992;4:410. 10.1016/0952-8180(92)90111-D
25. Kälsch H, Lehmann N, Möhlenkamp S, Becker A, Moebus S, Schmermund A, et al. Body-surface adjusted aortic reference diameters for improved identification of patients with thoracic aortic aneurysms: results from the population-based Heinz Nixdorf Recall study. Int J Cardiol. 2013;163:72-78. 10.1016/j.ijcard.2011.05. 039
Cite this article as: Villard C, Eriksson P, Swedenborg J, Hultgren R. Differences in Elastin and Elastolytic Enzymes between Men and Women with Abdominal Aortic Aneurysm. Aorta 2014;2(5): 179-185. DOI: http://dx.doi.org/10.12945/ j.aorta.2014.14-017 